\title{
In vitro screening of LPS-induced miRNAs in leukocytes derived from cord blood and their possible roles in regulating TLR signals
}

\author{
Jiande Chen ${ }^{1,2}$, Zhiwei Liu' ${ }^{1,2}$ and Yi Yang ${ }^{1,2}$
}

BACKGROUND: microRNAs (miRNAs) are involved in a wide variety of biological processes and play roles in the regulation of Toll-like receptor (TLR) signals. We hypothesized that lipopolysaccharide (LPS)-induced miRNAs in the leukocytes from cord blood (CB) play an important role in newborn innate immunity, specifically in the regulation of the TLR signaling pathway.

METHODS: The expression profiles of LPS-induced miRNAs and TLR-related genes were studied by microarray and PCR arrays, respectively. A bioinformatics analysis was used to identify the potential biological processes and targets involved in the TLR signals affected by these miRNAs.

RESULTS: A total of 85 miRNAs and 41 TLR-related genes were differentially expressed. The bioinformatics analysis showed that the potential target genes of these miRNAs were involved in regulation of cellular biosynthetic process, regulation of gene expression, regulation of macromolecule biosynthetic process, etc. Thirteen potential miRNA-mRNA interaction sites were found within the cDNA sequences of 11 differentially expressed TLR signaling pathway genes.

CONCLUSION: We identified a global miRNA expression signature occurring during LPS-induced acute inflammation in leukocytes derived from CB. The target genes were mainly involved in several biological processes, and these miRNAs may play important roles in the regulation of TLR signals. However, the precise mechanisms require further validation.

$\mathbf{T}$ he innate immune response mediated by immune cells involves the initial recognition of conserved pathogenassociated molecular patterns by members of the Toll-like receptor (TLR) family. Lipopolysaccharide (LPS), the main component of the outer membrane of Gram-negative bacteria, is the principal pathogen-associated molecular pattern of TLR4 (1). The immune system detects and responds to LPS via TLR4 and activates various transcription factors, leading to the strong production of proinflammatory cytokines (2). Sepsis, which leads to severe adverse outcomes such as organ dysfunction and death, can develop when the host defensive response to an infection is magnified (3). In human cord blood (CB) mononuclear cells, this LPS-triggered activation of the proinflammatory response results in many dysregulated genes that have been previously characterized by genome-wide expression profiling methods (4).

MicroRNAs (miRNAs) are a class of small noncoding RNAs that regulate gene expression either by the cleavage of mRNA targets or by the inhibition of protein translation. miRNAs act as key regulators in a wide variety of biological processes, including proliferation, differentiation, cell fate determination, apoptosis, signal transduction, and organ development, and abnormal miRNAs expression is a common feature of inflammatory diseases (5). Furthermore, the differential expression of miRNAs may help to distinguish between disease states (6). miRNAs have been identified as important regulators of immune responses (7) and as fine-tuners of TLRs (8). Multiple molecules involved in the TLR pathway are thought to be targeted by miRNAs, such as the expression of the TLRs themselves, TLR signal molecules, TLR-induced transcription factors, regulators of the TLR signaling pathway, and even the final functional cytokines involved in TLR signaling (9).

Although miRNAs (miR-18a and miR-155) have been proven to show significant expression changes in CB-derived $\mathrm{CD}_{14}{ }^{+}$cells in response to LPS (10) and the involvement of miR-146 in neonatal TLR4 signaling has been discussed to some extent (11), the global changes in the miRNA expression of leukocytes derived from CB during LPS activation and their biological and molecular mechanisms are still unclear. In this study, we will explore the LPS-induced miRNA expression profile in the cord leukocytes and speculate about the role of these miRNAs in newborn innate immunity, focusing specifically on TLR signaling pathway regulation.

\section{RESULTS}

LPS-Induced miRNA Expression Profiling

To identify the miRNAs involved in the regulation of the LPSinduced immune response, we used a commercial microarray chip that can examine the global expression level for each Homo sapiens miRNA annotated in miRBase 14.0. In 
leukocytes derived from newborn $\mathrm{CB}(n=3)$, we identified 85 miRNAs that were differentially expressed between the experimental and control groups, with 37 miRNAs being upregulated and 48 being downregulated (Table 1).

\section{Microarray-Based GO Analysis}

To assess the functions of these differentially expressed miRNAs, we performed computational predictions of the potential targets for these miRNAs via two algorithms (TargetScan and miRDB) and focused on those targets that were predicted by both. Gene Ontology (GO) analysis was applied to these putative target genes using DAVID (Database for Annotation, Visualization and Integrated Discovery). Interestingly, the most enriched GO terms for these target genes of the up- or downregulated miRNAs were extremely consistent. They were regulation of cellular biosynthetic process, regulation of gene expression, regulation of macromolecule biosynthetic process, regulation of nucleobase, nucleoside, nucleotide, and nucleic acid metabolic process, regulation of transcription, etc. (Figure 1a,b).

\section{LPS-Induced TLR-Related Genes Expression}

To determine the involvement of the TLRs in LPS treatment, 84 TLR-related genes were profiled by PCR array after an LPS challenge. LPS stimulation modulated the expression of 41 of the 84 genes examined; of those 41 genes, 28 were upregulated and 13 were downregulated compared with their expression levels in unstimulated cells ( $n=3$; fold change $>2$; Table 2 ). These differentially expressed genes in the TLR signaling pathway can be classified into several groups. (i) TLRs: TLR2 and TRL7 were upregulated, whereas TLR1, TLR5, TLR6, TLR8, and TLR9 were downregulated. (ii) TLR signaling. MyD88dependent: $M y D 88$ and IRAK2 were upregulated. Toll/ IL-1 receptor domain-containing adaptor inducing IFN$\boldsymbol{\beta}$-dependent: $T B K 1$ was upregulated. Negative regulation: SIGIRR was downregulated. (iii) Downstream pathways and target genes. Nuclear factor (NF)-кB pathway: IKBKB, REL, UBE2N, NFKB1, NFKBIA, and CHUK were upregulated, whereas $B T K, U B E 2 V 1$, and $N F R K B$ were downregulated. c-Jun N-terminal kinase/p38 pathway: FOS, MAP3K7IP1, and $M A P K 8 I P 3$ were downregulated. Janus kinase/signal transducer and activator of transcription pathway: CCL2, CSF2, and interleukin (IL)-6 were upregulated. IRF pathway: CXCL10, IFNA1, and IFNB1 were upregulated. Cytokinemediated signaling pathway: CSF3, IL-1A, IL-1B, and TNF were upregulated. (iv) Regulation of adaptive immunity: $C D 80$ and $I L-10$ were upregulated, whereas CD86 was downregulated. (v) Adaptors and TLR-interacting proteins: CLEC4E and TICAM1 were upregulated. (vi) Inflammatory mediators: LTA, IL-8, and PTGS2 were upregulated.

\section{Predicted Target Genes of LPS-Responsive miRNAs in the TLR Signaling Pathway}

To gain insight into the function of the LPS-responsive miRNAs in the TLR signaling pathway, we used TargetScan and miRDB to predict the potential targets in the TLR signaling pathway for these miRNAs and focused on miRNA-mRNA
Table 1. The list of differentially induced miRNAs in leukocytes derived from infant cord blood stimulated with LPS for $2 \mathrm{~h}$

\begin{tabular}{|c|c|c|c|}
\hline $\begin{array}{l}\text { miRNA name } \\
\text { (upregulated) }\end{array}$ & Fold change & $\begin{array}{c}\text { miRNA name } \\
\text { (downregulated) }\end{array}$ & Fold change \\
\hline hsa-miR-130a & 2.1 & hsa-let-7i & -2.8 \\
\hline hsa-miR-146b-5p & 3.2 & hsa-miR-107 & -2.3 \\
\hline hsa-miR-141 & 36.6 & hsa-miR-16 & -2.2 \\
\hline hsa-miR-142-3p & 3.0 & hsa-miR-182 & -48.8 \\
\hline hsa-miR-193a-3p & 2.8 & hsa-miR-183 & -7.8 \\
\hline hsa-miR-203 & 16.1 & hsa-miR-194 & -3.3 \\
\hline hsa-miR-214 & 10.4 & hsa-miR-215 & -2.2 \\
\hline hsa-miR-377 & 13.3 & hsa-miR-29b & -2.9 \\
\hline hsa-miR-200c & 8.7 & hsa-miR-29c & -3.3 \\
\hline hsa-miR-491-3p & 3.1 & hsa-miR-96 & -23.9 \\
\hline hsa-miRPlus-A1015 & 2.5 & hsa-miR-627 & -3.7 \\
\hline hsa-miR-199a-5p & 3.5 & hsa-miR-550a-3p & -3.1 \\
\hline hsa-miR-125b & 28.7 & hsa-miR-92b & -3.5 \\
\hline hsa-miR-129-5p & 3.0 & hsa-let-7b & -8.8 \\
\hline hsa-miR-933 & 3.0 & hsa-let-7f & -3.9 \\
\hline hsa-miR-326 & 3.6 & hsa-miR-106b-3p & -2.4 \\
\hline hsa-miR-34b & 2.0 & hsa-miR-500 & -2.7 \\
\hline hsa-miR-720 & 2.8 & hsa-miR-186 & -2.3 \\
\hline hsa-miR-181a & 3.2 & hsa-miR-190 & -4.9 \\
\hline hsa-miRPlus-E1104 & 3.1 & hsa-miR-574-3p & -2.0 \\
\hline hsa-miRPlus-E1088 & 2.1 & hsa-miR-486-5p & -2.1 \\
\hline hsa-miR-1246 & 4.1 & hsa-miR-126-5p & -4.6 \\
\hline hsa-miRPlus-E1117 & 2.4 & hsa-miR-576-5p & -5.1 \\
\hline hsa-miRPlus-F1195 & 2.0 & hsa-miR-362-5p & -4.4 \\
\hline hsa-miR-205 & 1407.1 & hsa-miR-582-5p & -6.6 \\
\hline hsa-miR-136 & 103.8 & hsa-miR-338-3p & -2.6 \\
\hline hsa-miR-142-5p & 2.8 & hsa-miR-20a-3p & -2.5 \\
\hline hsa-let-7c & 2.5 & hsa-miR-25 & -3.1 \\
\hline hsa-miR-210 & 4.1 & hsa-miR-943 & -3.1 \\
\hline hsa-miR-33a & 3.6 & hsa-miR-339-5p & -2.3 \\
\hline hsa-miR-494 & 2.8 & hsa-miR-197 & -2.0 \\
\hline hsa-miR-27b & 2.4 & hsa-miR-652 & -2.6 \\
\hline hsa-miR-33b & 4.2 & hsa-miR-451 & -3.2 \\
\hline hsa-miR-200b & 125.0 & hsa-miR-16-2-3p & -9.8 \\
\hline hsa-miR-1260 & 3.6 & hsa-miRPlus-E1285 & -2.2 \\
\hline hsa-miR-3202 & 10.5 & hsa-miRPlus-F1181 & -2.1 \\
\hline \multirow[t]{12}{*}{ hsa-miR-1973 } & 7.8 & hsa-miRPlus-E1245 & -71.9 \\
\hline & & hsa-miR-1827 & -2.3 \\
\hline & & hsa-miR-1297 & -5.5 \\
\hline & & hsa-miR-29a-5p & -2.5 \\
\hline & & hsa-let-7i-3p & -4.2 \\
\hline & & hsa-miR-484 & -2.4 \\
\hline & & hsa-miR-374a & -2.6 \\
\hline & & hsa-miR-20b & -2.7 \\
\hline & & hsa-let-7d & -3.3 \\
\hline & & hsa-let-7a & -2.7 \\
\hline & & hsa-miR-502-3p & -3.3 \\
\hline & & hsa-miR-103a-2-5p & -3.9 \\
\hline
\end{tabular}

LPS, lipopolysaccharide; miRNA, microRNA. 


\section{LPS-induced miRNAs in TLR signals Articles}

a

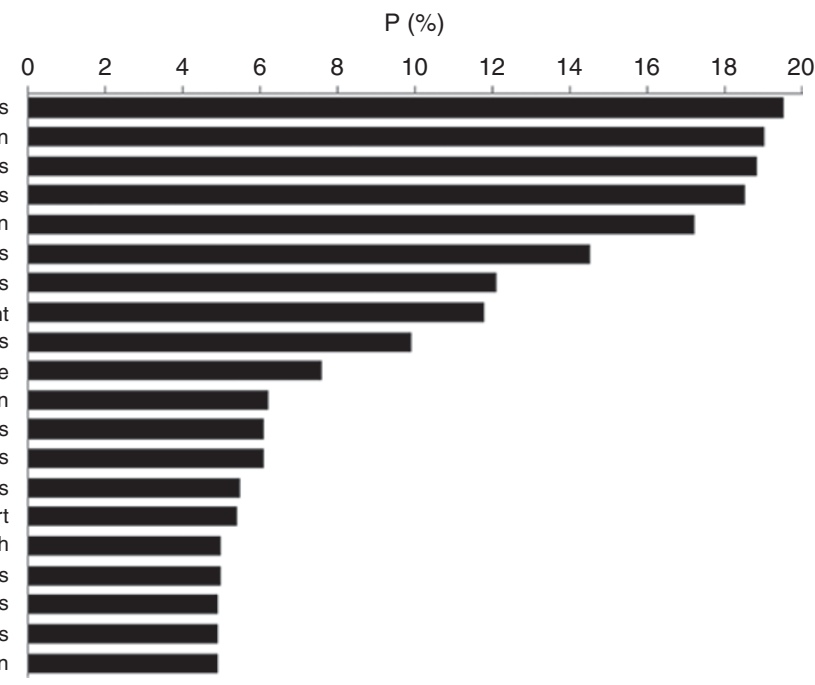

b

Regulation of macromolecule biosynthetic process

Regulation of nucleobase, nucleoside, nucleotide, and NA metabolic process

Cellular protein metabolic process

Regulation of RNA metabolic process Regulation of transcription, DNA-dependen Protein modification process Intracellular signaling cascad Regulation of signal transduction Positive regulation of macromolecule metabolic process Positive regulation of cellular metabolic process RNA metabolic process Protein transpor

Regulation of programmed cell death Regulation of apoptosis Positive regulation of biosynthetic process Positive regulation of cellular biosynthetic process Phosphorylation

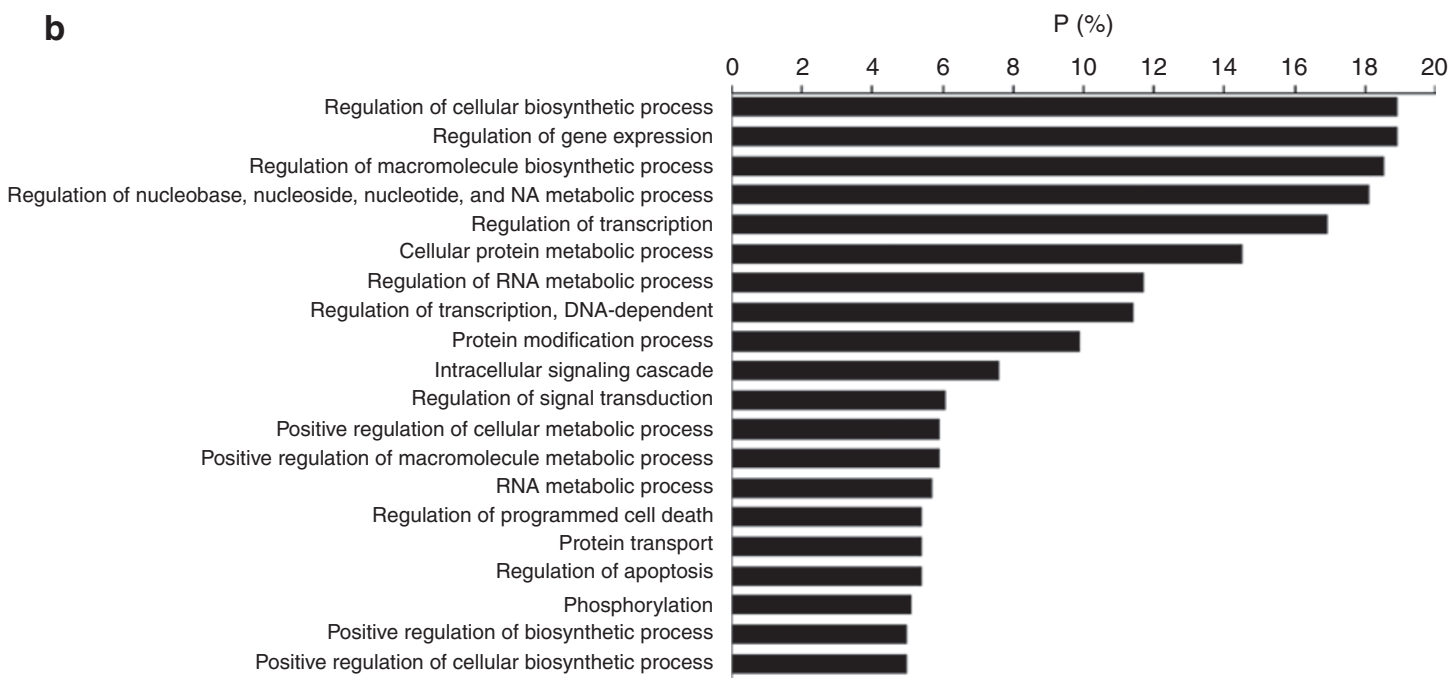

Figure 1. Gene Ontology (GO) category for putative target genes. (a) and (b) show the significant GOs of differential genes targeted by the upregulated and downregulated miRNAs, respectively. $\mathrm{P}$ is the percentage of predicted target genes, and larger P values indicate higher levels of enrichment. NA, nucleic acid.

pairs that were coexpressed in leukocytes. A total of 13 potential miRNA-mRNA interaction sites for 10 differentially expressed miRNAs were found within the cDNA sequences of 11 differentially expressed TLR signaling pathway genes (Table 3).

\section{Quantitative Real-Time PCR Verification of miRNA Array Results}

To confirm the results of the miRNA array, we selected six miRNAs from Table 3 for further validation in another set of samples from different subjects $(n=3)$. The quantitative realtime (RT)-PCR results showed that the miRNAs were up- or downregulated in a manner consistent with the miRNA array results (Figure 2). MiR-129-5p and miR-1260 were upregulated in the LPS-stimulated group, whereas miR-183, miR-186, miR-339-5p, and miR-374a were downregulated.

\section{DISCUSSION}

In the present study, we analyzed the miRNA expression profiles in the leukocytes derived from newborn $\mathrm{CB}$ after $2 \mathrm{~h}$ of
LPS stimulation. We found 85 miRNAs, with 37 being upregulated and 48 being downregulated after LPS stimulation. These findings indicated that the response to LPS might induce rapid and specific in vitro changes to the miRNA expression profile of leukocytes derived from CB and that these miRNAs might play important roles in the neonatal immune response. However, some miRNAs, including miR-150, miR-342, let$7 \mathrm{~g}$, and miR-143, which have been shown to be differentially expressed in LPS-stimulated leukocytes derived from adult venous blood (12), were not changed in the LPS-stimulated leukocytes derived from CB; in addition, miR-146b, which was downregulated in adults, was upregulated in the CB, indicating that miRNAs might respond differently in LPS-stimulated leukocytes in newborns than in adults and play contrastingly different roles between them.

Among the differentially expressed miRNAs, miR-205, miR136, miR-200b, miR-141, and miR-182 were highly deregulated. Most of these miRNAs have been shown to be involved in both normal development and cancer. The miR-200 family 


\section{Articles | Chen et al.}

Table 2. LPS-induced gene expression in TLR signaling pathway

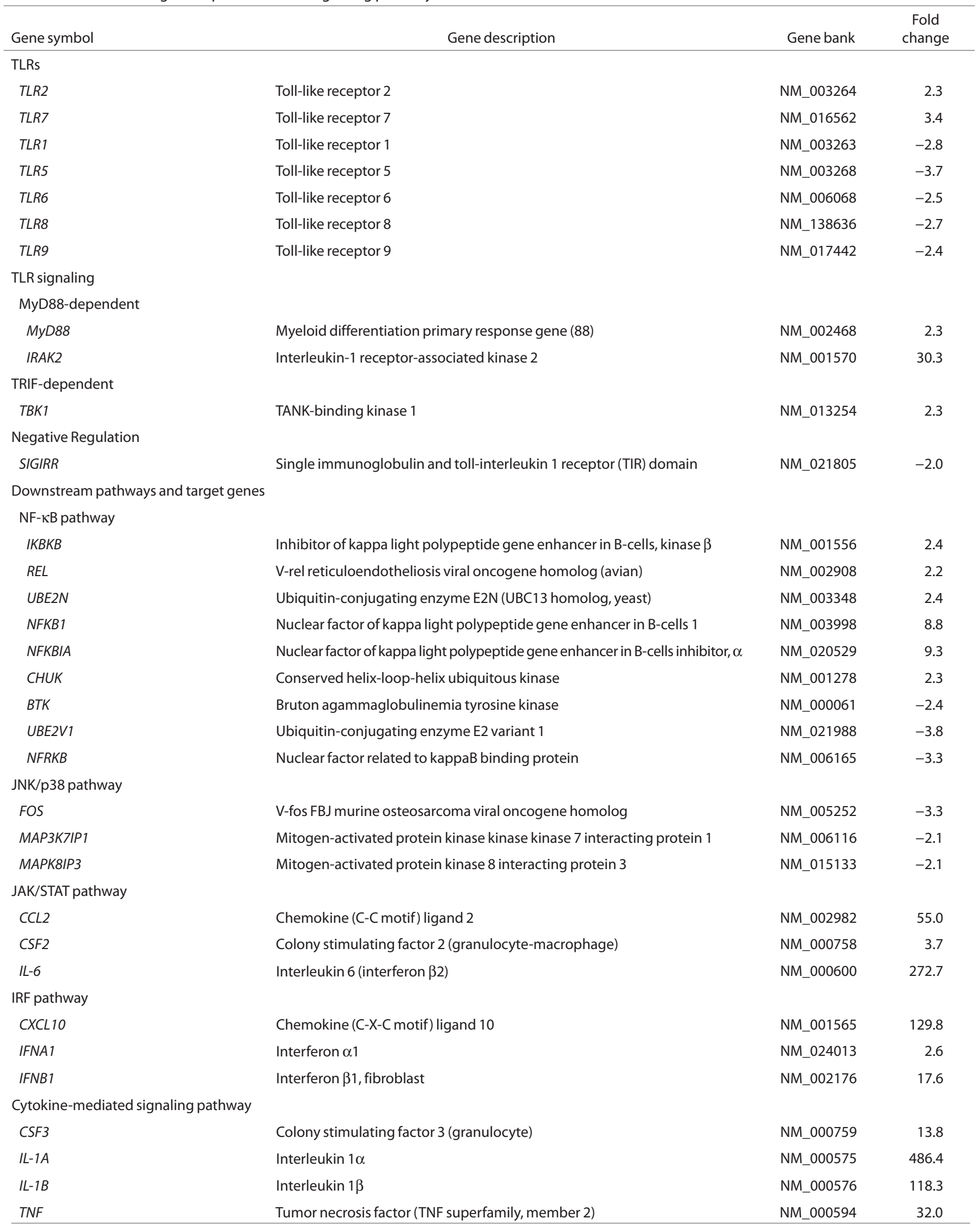

Table 2. Continued on next page. 
Table 2. (Continued)

\begin{tabular}{|c|c|c|c|}
\hline Gene symbol & Gene description & Gene bank & $\begin{array}{l}\text { Fold } \\
\text { change }\end{array}$ \\
\hline \multicolumn{4}{|c|}{ Regulation of adaptive immunity } \\
\hline IL-10 & Interleukin 10 & NM_000572 & 3.5 \\
\hline CD86 & CD86 molecule & NM_006889 & -2.5 \\
\hline \multicolumn{4}{|c|}{ Adaptors and TLR-interacting proteins } \\
\hline TICAM1 & Toll-like receptor adaptor molecule 1 & NM_182919 & 7.0 \\
\hline \multicolumn{4}{|c|}{ Inflammatory mediators } \\
\hline LTA & Lymphotoxin $\alpha$ (TNF superfamily, member 1$)$ & NM_000595 & 4.3 \\
\hline IL-8 & Interleukin 8 & NM_000584 & 20.9 \\
\hline PTGS2 & $\begin{array}{l}\text { Prostaglandin-endoperoxide synthase } 2 \text { (prostaglandin G/H synthase } \\
\text { and cyclooxygenase) }\end{array}$ & NM_000963 & 3.1 \\
\hline
\end{tabular}

JAK/STAT, Janus kinase/signal transducer and activator of transcription; JNK, c-Jun N-terminal kinase; LPS, lipopolysaccharide; NF, nuclear factor; TLR, Toll-like receptor; TRIF, Toll//L-1 receptor domain-containing adaptor inducing IFN- $\beta$.

(miR-200a, miR-200b, miR-200c, miR-141, and miR-429) and miR-205 are frequently silenced in advanced cancer. A study on mammary gland stem or progenitor cells showed that miR205 overexpression led to an expansion of the progenitor-cell population, decreased cell size, and increased cellular proliferation (13). However, there is no report on the effect of the miR200 family on immune cells. In our study, the higher expression levels of miR-200b, miR-141, and miR-205 may be related to the increased number of stem cells in the CB. As an important immune regulator, miR-182 has been demonstrated to have a central role in the physiological regulation of the IL-2-driven helper T-cell-mediated immune response (14). Therefore, the downregulation of miR-182 might have special significance for LPS-induced immune response. However, although these miRNAs were obviously deregulated in LPS-stimulated leukocytes derived from $\mathrm{CB}$, their immune functions in newborn inflammation must be carefully interpreted.

To gain significant insight into the function of these LPSinduced differentially expressed miRNAs, their target genes were predicted, and GO analysis was applied to these predicted target genes. The GO categories showed that the biological processes regulated by the differentially expressed miRNAs mainly included regulation of cellular biosynthetic process, regulation of gene expression, regulation of macromolecule biosynthetic process, regulation of nucleobase, nucleoside, nucleotide, and nucleic acid metabolic process, regulation of transcription, etc. for both the up- and downregulated miRNAs. This finding indicated that these miRNAs might act together on the same biological processes to control the robust proinflammatory response in the acute inflammatory state induced by LPS stimulation. Additionally, the regulation of gene expression and transcription by target genes could be an indirect mechanism to help the miRNAs strengthen this control.

In contrast with the study that used cRNA microarray technology to identify differentially expressed genes involved in the LPS-stimulated response of mononuclear cells from CB on a "global" level (4) and the study that detected certain factors
Table 3. Target genes of LPS-responsive miRNAs in TLR signaling pathway

\begin{tabular}{ll}
\hline miRNA & Gene symbol \\
\hline $\begin{array}{l}\text { Upregulated } \\
\text { hsa-miR-129-5p }\end{array}$ & CD86 \\
hsa-miR-494 & TLR6 \\
hsa-miR-1260 & CD86 \\
Downregulated & \\
hsa-miR-16 & CD80 \\
hsa-miR-183 & NFKB1, PTGS2 \\
hsa-miR-186 & UBE2N \\
hsa-miR-339-5p & CSF3, IRAK2 \\
hsa-miR-374a & $C C L 2$, PTGS2 \\
hsa-miR-500a & IL-8 \\
hsa-miR-1827 & LTA \\
\hline
\end{tabular}

LPS, lipopolysaccharide; miRNA, microRNA; TLR, Toll-like receptor.

related to the LPS-induced TLR pathway response in the monocytes derived from CB (11), the aim of this exploratory study was to determine which genes in the LPS-stimulated leukocytes from CB that are associated with TLRs and their signaling elements were differentially expressed on the whole. In our study, 41 of the 84 genes examined were found to be differentially expressed in TLR signaling pathways between leukocytes from CB with or without LPS stimulation. Via the MyD88-dependent pathway, which induces NF- $\kappa B-$ dependent proinflammatory cytokines (IL- $1 \alpha$, IL-1 $\beta$, IL-6, IL-8, and TNF- $\alpha$ ), and the MyD88-independent pathway, which induces Toll/IL-1 receptor domain-containing adaptor inducing IFN$\beta$-dependent interferon-inducible cytokines $(15,16)$, LPSstimulated leukocytes from CB showed a predominant initial increase in the production of inflammatory cytokines.

The components of TLR signaling can be directly targeted by miRNAs. For example, the mRNA encoding MyD88, which links members of the TLR family to the downstream 


\section{Articles $\mid$ Chen et al.}

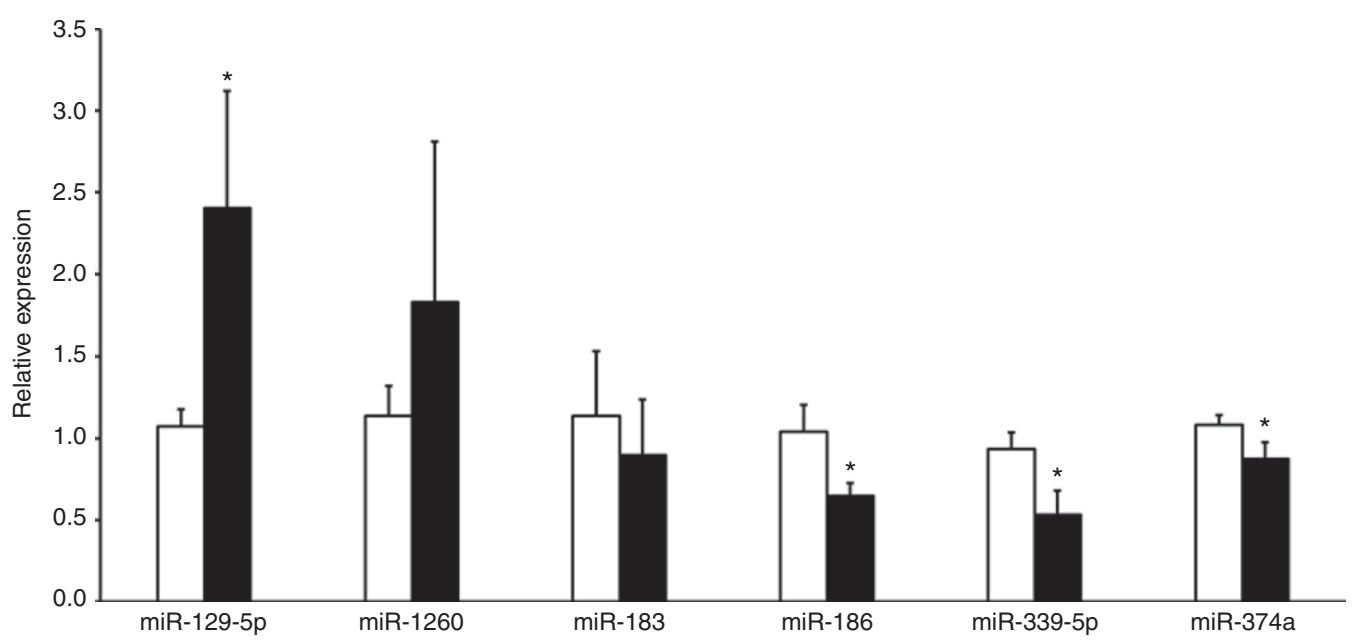

Figure 2. Selected microRNA (miRNA) array results were confirmed by real-time PCR in another set of samples from different subjects ( $n=3$ ). The expression of six miRNAs in lipopolysaccharide-stimulated group (black bar) was compared with that of the control group (white bar). The relative expression values are the normalized mean \pm SD. ${ }^{*} P<0.05$ by the $t$-test.

activation of NF- $\mathrm{KB}$ and mitogen-activated protein kinases (17), could be directly repressed by miR-200b and miR200c (18). Proinflammatory IL-6 and TNF are direct targets of Let-7a (19) and miR-16 (20), respectively. We think that the inhibition of the interaction between the LPS-induced miRNAs and their targets and/or insufficient expression of these miRNAs that target TLR signaling might contribute to the robust proinflammatory response in the acute inflammatory state induced by LPS stimulation. Further studies will be needed to evaluate these issues.

miRNA expression can be directly regulated by TLR signals. For example, miR-181a was found to be concurrently upregulated with IL-1 $\alpha$, IL-1 $\beta$, IL-6, and TNF- $\alpha$ in a mouse acute inflammatory state via a TLR4-dependent pathway (21). MiR-107, which was downregulated in response to LPS in multiple cell types (22), and let-7i, which was decreased in LPSstimulated cholangiocytes (23), were found to be affected in a MyD88- and NF- $\kappa B-$ dependent manner. Similar coexpression patterns between these miRNAs and TLR signaling compartments in our study might support those results. The interaction between miRNAs and the TLR signaling pathway reveals a feedback loop that controls TLR signaling activation (24).

In LPS-stimulated THP-1 cells, upregulated miR-146 has been proposed to play a role in the control of TLR and cytokine signaling through a negative feedback regulation loop (25). The upregulation of miR-146 after LPS stimulation was also observed in monocytes derived from adult peripheral veins and from newborn CB (11). After LPS engagement, monocytes upregulate miR-146b which modulates the TLR4 signaling pathway by direct targeting of multiple elements, including TLR4, MyD88, IRAK-1, and TRAF6, via an IL-10mediated STAT3-dependent loop (26). In addition, activation of the MAP kinase/EGR pathway by proinflammatory cytokines regulates the transcription of the miR-146, which can in turn repress the NF- $\mathrm{\kappa B}, \mathrm{AP}-1$, and MAPK/EGR pathways. Thus, a negative feedback loop is formed to control the proinflammatory signaling in endothelial cells that may impact the pathogenesis of vascular inflammatory diseases such as sepsis (27). In our study, the expression of miR-146b was upregulated in CB leukocytes after LPS stimulation, indicating that miR-146b might have an important role in controlling LPSstimulated neonatal early phases of inflammation by engaging in negative feedback loops.

The prediction analyses of the miRNA-mRNA pairs that are coexpressed in leukocytes, which may offer intriguing new perspectives on the interaction between miRNA regulation and TLR signals, identified 11 different candidate genes in the TLR signaling pathway for degradation control by miRNAs. PTGS2 - a candidate target of the LPS-decreased miR-183 and miR-374a - was upregulated in leukocytes during LPS-induced inflammation. PTGS2 is considered a proinflammatory mediator during the early phases of inflammation and a chief target for the treatment of inflammatory diseases (28). Hence, the decrease in miR-183 and miR-374a could play a role in supporting the upregulation of PTGS2, which may be partially responsible for the upregulation of some cytokines in our model.

This preliminary study has potential limitations. First, an in vitro study of whole blood may not reflect the patterns of response in vivo. Second, our study did not conduct functional studies in cells to validate the predicted mRNA targets. Third, the whole blood stimulation analysis limits the conclusions on cell-specific mechanisms involving interactions between miRNA regulation and TLR signaling. Further studies are necessary to determine whether these differentially expressed miRNAs regulate each of these predicted target genes and how these miRNAs influence the development of neonatal sepsis.

In conclusion, we identified differentially expressed miRNAs in the LPS-induced acute inflammation of leukocytes derived from $\mathrm{CB}$ and performed a functional bioinformatic analysis that demonstrated that the target genes regulated by these miRNAs were mainly involved in several biological processes. In addition, these miRNAs may play important roles in the regulation of TLR signals. Further investigation is needed to validate the involvement of these miRNAs in neonatal sepsis. 


\section{METHODS}

\section{Blood Sampling}

CB samples were obtained by sterile puncture of the umbilical cords of six healthy, full-term infants delivered at the Obstetrics Department of the International Peace Maternity and Child Health Hospital of China Welfare Institute, Shanghai after obtaining informed consent from the parent(s). The newborns exhibited no signs of bacterial infection during a follow-up of $1 \mathrm{wk}$. Blood was collected in lithium heparin collection tubes and processed within $2 \mathrm{~h}$ after collection. The study was performed in accordance with the policies of and approved by the Ethics Committee of Children's Hospital of Fudan University, Shanghai, China.

\section{Whole Blood Cell Culture and LPS Stimulation}

The blood samples were diluted 1:1 with RPMI 1640 culture medium supplemented with penicillin $(100 \mathrm{IU} / \mathrm{ml})$ and streptomycin $(0.1 \mathrm{mg} /$ $\mathrm{ml}$ ) and immediately transferred as 6-ml aliquots into two pyrogen-free $15-\mathrm{ml}$ Corning tubes. The diluted whole neonatal $\mathrm{CB}$ with (experimental group) or without (control group) $1 \mu \mathrm{g} / \mathrm{ml}$ Escherichia coli LPS (Sigma-Aldrich, St. Louis, MO) was incubated at $37^{\circ} \mathrm{C}$ in a humidified chamber with $5 \% \mathrm{CO}_{2}$ for $2 \mathrm{~h}$.

\section{Leukocyte Isolation and Total RNA Extraction}

A 6-ml aliquot of whole blood culture was mixed with $1.2 \mathrm{ml}$ of $6 \%$ dextran (Pharmacia, Uppsala, Sweden) solution in saline. After sedimentation for $30 \mathrm{~min}$ at $37^{\circ} \mathrm{C}$, the leukocyte-enriched supernatant was collected and washed twice with saline before the RNA isolation. Total RNA was isolated from leukocytes using TRIzol reagent (Invitrogen, Carlsbad, CA) according to the manufacturer's instructions. DNA contamination was removed from the RNA preparations using DNase I (Qiagen, Hilden, Germany) followed by an RNA cleanup kit (RNeasy MinElute Cleanup Kit, Qiagen). The total RNA concentration and purity were determined by the NanoDrop ND-1000 (Thermo, Wilmington, DE). Electrophoresis (1\% agarose gel containing ethidium bromide) was used to confirm the total RNA integrity. Only RNA samples passing the purity (OD260/280: 1.8-2.1) and integrity (28S:18S bands in a ratio of $\sim 2: 1$ with a clear and trailfree manner and no nonspecific bands) controls were used for further miRNA microarray and reverse transcription PCR analyses.

\section{miRNA Array}

Equal amounts of RNA from three different samples were pooled for each experimental condition and then labeled using the miRCURY Hy3/Hy5 Power labeling kit (Exiqon, Vedbaek, Denmark). The labeled RNA was hybridized on the miRCURY LNA Array (v.14.0) (Exiqon). Scanning was performed with the Axon GenePix 4000B microarray scanner (Axon, Foster, CA). The GenePix pro V6.0 software (Axon) was used to read the raw intensity of the image. The ratio of green to red signals was calculated after background subtraction and normalization using the global Lowess (Locally Weighted Scatter plot Smoothing) regression algorithm (MIDAS, TIGR Microarray Data Analysis System software; J. Craig Venter Institute, Rockville, $\mathrm{MD}$ and La Jolla, CA). The threshold value used to screen the up- and downregulated miRNAs was a fold change of $>2.0$.

\section{Quantitative RT-PCR}

Quantitative RT-PCR was used to confirm the miRNA array results. Briefly, 10 ng of total RNA was reverse-transcribed using the TaqMan miRNA reverse transcription kit with the primers for miR-129-5p, miR-1260, miR-183, miR-186, miR-339-5p, miR-374a, and U6 small nuclear RNA (Applied Biosystems, Foster, CA). Then, the TaqMan probes for these miRNAs were used in the following real-time PCRs. The relative expression of each miRNA was determined in reference to an internal U6 small nuclear RNA control. In the statistical analysis, we presented the results as the mean \pm SD and assessed the differences between the groups with the independent $t$-test using SPSS software 16.0 (SPSS, Chicago, IL).

Quantitative RT-PCR arrays were used to detect the expression of the TLR pathway genes. A total of $1.5 \mu \mathrm{g}$ of RNA was used for cDNA synthesis using SuperScript III Reverse Transcriptase (Invitrogen). Real-time PCR was performed using the 384-well RT ${ }^{2}$ Profiler PCR Array (SuperArray Bioscience, Frederick, MD). A total of 84 genes involved in the human TLR pathway (full details are provided in
Supplementary Table S1) were analyzed for each sample. Two genes (B2M and RPL13A) were used as internal controls, and the average of their cycle threshold $(\mathrm{Ct})$ values was used to normalize the gene expression $\left(2^{-\Delta \mathrm{Ct}}\right)$ and determine the fold change between groups $\left(2^{-\Delta \Delta C t}\right)$. Gene expression was considered up- or downregulated when the fold change was greater than 2 .

\section{Target Gene Prediction and Functional Analysis}

To predict the target genes of differentially expressed miRNAs, two online search algorithms, TargetScan Release 6.2 database (Whitehead Institute for Biomedical Research, Cambridge, MA) and miRDB Version 4.0 database (Department of Radiation Oncology, Washington University School of Medicine, St. Louis, MO) (29,30), were used for target prediction. The genes that were identified by both the algorithms were considered to be the target genes. To further understand the functions of the predicted target genes, we used the ontology classification of genes based on gene annotation and summary information available through DAVID Bioinformatics Resources 6.7 database (Laboratory of Immunopathogenesis and Bioinformatics, SAIC-Frederick, Frederick, MD) $(31,32)$ to perform a preliminary analysis of the forecasted target genes.

\section{SUPPLEMENTARY MATERIAL}

Supplementary material is linked to the online version of the paper at http:// www.nature.com/pr

\section{ACKNOWLEDGMENTS}

The authors thank the International Peace Maternity and Child Health Hospital of China Welfare Institute, Shanghai, for its help.

\section{STATEMENT OF FINANCIAL SUPPORT}

This study was supported by the National Natural Science Foundation of China (grant no. 81070518).

Disclosure: The authors report no conflicts of interest.

\section{REFERENCES}

1. Song DH, Lee JO. Sensing of microbial molecular patterns by Toll-like receptors. Immunol Rev 2012;250:216-29.

2. Nahid MA, Satoh M, Chan EK. MicroRNA in TLR signaling and endotoxin tolerance. Cell Mol Immunol 2011;8:388-403.

3. Warren HS. Strategies for the treatment of sepsis. N Engl J Med 1997;336:952-3.

4. Koch L, Linderkamp O, Ittrich C, Benner A, Poeschl J. Gene expression profiles of adult peripheral and cord blood mononuclear cells altered by lipopolysaccharide. Neonatology 2008;93:87-100.

5. Sonkoly E, Ståhle M, Pivarcsi A. MicroRNAs and immunity: novel players in the regulation of normal immune function and inflammation. Semin Cancer Biol 2008;18:131-40.

6. Perron MP, Boissonneault V, Gobeil LA, Ouellet DL, Provost P. Regulatory RNAs: future perspectives in diagnosis, prognosis, and individualized therapy. Methods Mol Biol 2007;361:311-26.

7. Baltimore D, Boldin MP, O'Connell RM, Rao DS, Taganov KD. MicroRNAs: new regulators of immune cell development and function. Nat Immunol 2008;9:839-45.

8. O'Neill LA, Sheedy FJ, McCoy CE. MicroRNAs: the fine-tuners of Toll-like receptor signalling. Nat Rev Immunol 2011;11:163-75.

9. Li Y, Shi X. MicroRNAs in the regulation of TLR and RIG-I pathways. Cell Mol Immunol 2013;10:65-71

10. Takahashi N, Nakaoka T, Yamashita N. Profiling of immune-related microRNA expression in human cord blood and adult peripheral blood cells upon proinflammatory stimulation. Eur J Haematol 2012;88:31-8.

11. Lederhuber H, Baer K, Altiok I, Sadeghi K, Herkner KR, Kasper DC. MicroRNA-146: tiny player in neonatal innate immunity? Neonatology 2011;99:51-6.

12. Schmidt WM, Spiel AO, Jilma B, Wolzt M, Müller M. In vivo profile of the human leukocyte microRNA response to endotoxemia. Biochem Biophys Res Commun 2009;380:437-41.

13. Greene SB, Herschkowitz JI, Rosen JM. The ups and downs of miR-205: identifying the roles of miR-205 in mammary gland development and breast cancer. RNA Biol 2010;7:300-4. 


\section{Articles | Chenetal.}

14. Stittrich AB, Haftmann C, Sgouroudis E, et al. The microRNA miR-182 is induced by IL-2 and promotes clonal expansion of activated helper T lymphocytes. Nat Immunol 2010;11:1057-62.

15. Akira S, Takeda K. Toll-like receptor signalling. Nat Rev Immunol 2004;4:499-511.

16. Yamamoto M, Sato S, Hemmi H, et al. Role of adaptor TRIF in the MyD88independent toll-like receptor signaling pathway. Science 2003;301:640-3.

17. Janssens S, Beyaert R. A universal role for MyD88 in TLR/IL-1R-mediated signaling. Trends Biochem Sci 2002;27:474-82.

18. Wendlandt EB, Graff JW, Gioannini TL, McCaffrey AP, Wilson ME. The role of microRNAs miR-200b and miR-200c in TLR4 signaling and NF-?B activation. Innate Immun 2012;18:846-55.

19. Iliopoulos D, Hirsch HA, Struhl K. An epigenetic switch involving NFkappaB, Lin28, Let-7 MicroRNA, and IL6 links inflammation to cell transformation. Cell 2009;139:693-706.

20. Jing Q, Huang S, Guth S, et al. Involvement of microRNA in AU-rich element-mediated mRNA instability. Cell 2005;120:623-34.

21. Xie W, Li Z, Li M, Xu N, Zhang Y. miR-181a and inflammation: miRNA homeostasis response to inflammatory stimuli in vivo. Biochem Biophys Res Commun 2013;430:647-52.

22. Hennessy EJ, Sheedy FJ, Santamaria D, Barbacid M, O’Neill LA. Toll-like receptor-4 (TLR4) down-regulates microRNA-107, increasing macrophage adhesion via cyclin-dependent kinase 6. J Biol Chem 2011;286:25531-9.

23. Chen XM, Splinter PL, O'Hara SP, LaRusso NF. A cellular micro-RNA, let-7i, regulates Toll-like receptor 4 expression and contributes to cholangiocyte immune responses against Cryptosporidium parvum infection. J Biol Chem 2007;282:28929-38.

24. Olivieri F, Rippo MR, Prattichizzo F, et al. Toll like receptor signaling in “inflammaging": microRNA as new players. Immun Ageing 2013;10:11.

25. Taganov KD, Boldin MP, Chang KJ, Baltimore D. NF-kappaB-dependent induction of microRNA miR-146, an inhibitor targeted to signaling proteins of innate immune responses. Proc Natl Acad Sci USA 2006;103:12481-6.

26. Curtale G, Mirolo M, Renzi TA, Rossato M, Bazzoni F, Locati M. Negative regulation of Toll-like receptor 4 signaling by IL-10-dependent microRNA146b. Proc Natl Acad Sci USA 2013;110:11499-504.

27. Cheng HS, Sivachandran N, Lau A, et al. MicroRNA-146 represses endothelial activation by inhibiting pro-inflammatory pathways. EMBO Mol Med 2013;5:949-66.

28. Vane J. Towards a better aspirin. Nature 1994;367:215-6.

29. Wang X, El Naqa IM. Prediction of both conserved and nonconserved microRNA targets in animals. Bioinformatics 2008;24:325-32.

30. Wang X. miRDB: a microRNA target prediction and functional annotation database with a wiki interface. RNA 2008;14:1012-7.

31. Huang da W, Sherman BT, Lempicki RA. Systematic and integrative analysis of large gene lists using DAVID bioinformatics resources. Nat Protoc 2009;4:44-57.

32. Huang da W, Sherman BT, Lempicki RA. Bioinformatics enrichment tools: paths toward the comprehensive functional analysis of large gene lists. Nucleic Acids Res 2009;37:1-13. 